\title{
Factores inmunogenéticos asociados al desarrollo de endometriosis
}

\author{
Alfredo Cortes-Algara', Julio Granados², Juan A. Pineda-Juaréz ${ }^{3}$, Juan A. Suárez-Cuenca4, \\ Daniel Santillán-Cortez ${ }^{3}$, Paul Mondragón-Terán ${ }^{3}$ y Mónica Escamilla-Tilch ${ }^{3 *}$
}

${ }^{1}$ Servicio de Ginecología, Centro Médico Nacional 20 de Noviembre, Instituto de Seguridad y Servicios Sociales de los Trabajadores del Estado; ${ }^{2}$ Departamento de Trasplantes, División de Inmunogenética, Instituto Nacional de Ciencias Médicas y Nutrición Salvador Zubirán; ${ }^{3}$ Coordinación de Investigación, Centro Médico Nacional 20 de Noviembre, Instituto de Seguridad y Servicios Sociales de los Trabajadores del Estado; ${ }^{4}$ Servicio de Investigación Clínica, Centro Médico Nacional 20 de Noviembre, Instituto de Seguridad y Servicios Sociales de los Trabajadores del Estado. Ciudad de México, México

\section{Resumen}

La endometriosis es una enfermedad proinflamatoria heredable, caracterizada por la presencia de glándulas y estroma endometrial funcionales fuera de la cavidad uterina, en la cual existe una desregulación en los componentes inmunológicos contenidos en el líquido peritoneal y un incremento de macrófagos activados, disminución de la inmunidad celular, inhibición citotóxica de las células natural killer (NK) y la activación de linfocitos B. La supervivencia de las células endometriales ectópicas se debe a la evasión de lisis al modular la expresión de moléculas de complejo mayor de histocompatibilidad clase l; aunado a esto, el microambiente generado por las citocinas regula la progresión de la endometriosis, sobreexpresando citocinas como interleucina (IL) 1, IL-6, factor de necrosis tumoral alfa e IL-8, que favorecen el infiltrado celular, depósitos de colágeno y angiogénesis; pero, al incrementar los niveles de IL-10, IL-6 y factor de crecimiento transformante beta se favorece la regulación sobre los linfocitos $B$, linfocitos $T$ y NK. En diversos estudios se han propuesto marcadores genéticos involucrados en la modulación de moléculas importantes que participan durante la respuesta inmunitaria; entre los más importantes están los genes del sistema HLA (antígenos leucocitarios humanos), los genes de los KIR (immuglobulin-like receptor) y los de los SNP (polimorfismos de nucleótido único, single nucleotide polymorphisms), lo que podría contribuir a la susceptibilidad y/o desarrollo de la patología.

Palabras clave: Endometriosis. HLA. KIR. SNP.

\section{Immunogenetic factors associated with the development of endometriosis}

\begin{abstract}
Endometriosis is a heritable proinflammatory disease characterized by the presence of functional endometrial glands and stroma outside the uterine cavity by a deregulation of the immunological components contained in the peritoneal fluid and an increase in activated macrophages, a decrease in cellular immunity, cytotoxic inhibition of NK cells and the activation of $B$ lymphocytes. The survival of ectopic endometrial cells is due to the avoidance of lysis by modulating the expression of MHC class I molecules; in addition to this, the microenvironment generated by the cytokines regulate the progression of endometriosis, overexpressing cytokines such as IL-1, IL-6, TNF- $\alpha$ and IL-8 that confers advantage of cell infiltration, collagen deposits and angiogenesis; but, by increasing the levels of IL-10, IL-6 and TGF- $\beta$, regulation on B lymphocytes, $T$ lymphocytes and NKs is favored. Several studies have proposed genetic markers involved in the modulation of important molecules that participate during the immune response, such as the HLA genes and the KIR genes and SNPs into genes to cytokines; which could contribute to the susceptibility and/or development of the pathology.
\end{abstract}

Key words: Endometriosis. HLA. KIRs. SNPS.

Correspondencia:

*Mónica Escamilla-Tilch

E-mail: monica.escamilla@issste.gob.mx
Fecha de recepción: 04-03-2020

Fecha de aceptación: 01-03-2021

DOI: 10.24875/REMQ.20000006
Disponible en internet: 04-06-2021

Rev Esp Méd Quir. 2020;25:71-81 www.remq-issste.com

1665-7330 / @ 2021 Revista de Especialidades Médico-Quirúrgicas. Publicado por Permanyer México SA de CV. Este es un artículo Open Access bajo la licencia CC BY-NC-ND (http://creativecommons.org/licenses/by-nc-nd/4.0/). 


\section{Introducción}

La endometriosis es una condición común, crónica y potencialmente debilitante que afecta a mujeres en edad reproductiva. Es una enfermedad proinflamatoria, dependiente de estrógeno, caracterizada por la presencia de glándulas y estroma endometrial funcionales fuera de la cavidad uterina. Los sitios más frecuentes de implante son el peritoneo pélvico, ovarios, ligamentos uterosacros y saco de Douglas'.

Los síntomas pueden variar, pero típicamente incluyen dismenorrea, dolor pélvico no relacionado con el ciclo menstrual, dispareunia, síntomas urinarios no especificados e infertilidad ${ }^{2}$. En México se estima una incidencia del $34 \%$ en mujeres con infertilidad, presentando el $50 \%$ de ellas una endometriosis leve, por lo que se ubica como una de las tres principales causas de atención ginecológica ${ }^{3}$.

El diagnóstico histopatológico requiere la presencia de al menos dos de las siguientes características: epitelio endometrial o glándulas endometriales fuera de la zona endometrial, estroma endometrial y/o macrófagos hemosiderados 4 .

La etiología de la endometriosis es compleja y multifactorial. Se han propuesto varias teorías para describir la patogénesis, entre las cuales están: a) la menstruación retrógrada, b) metaplasia endometrial, c) hiperestrogenismo, d) supresión de la apoptosis en células endometriales ectópicas, e) generación de tejido endometrial ectópico a partir de células madre, f) desarrollo de inflamación, producción de estrés oxidativo, falla en la inmunovigilancia y resolución de la respuesta inmunitaria, y g) una susceptibilidad genética que favorece el desarrollo de la endometriosis $^{5}$.

La menstruación retrógrada es el mecanismo más aceptado y consiste en la diseminación e implantación de epitelio uterino y células estromales en la cavidad peritoneal a través de las trompas de Falopio ${ }^{6}$. Sin embargo, el $90 \%$ de las mujeres presentan menstruación retrógrada y solamente un 6 a 10\% desarrollan la enfermedad ${ }^{7}$. Esta discrepancia confirma que en el desarrollo de endometriosis actúan distintos factores genéticos, bioquímicos y/o inmunológicos ${ }^{6}$.

Otro factor importante en la implantación y la proliferación de la endometriosis es la regulación del crecimiento celular ectópico, el cual está influenciado por una susceptibilidad genética y por una respuesta inmunitaria específica de cada individuo $0^{8,9}$.

\section{Fisiopatología de la endometriosis}

En la fisiopatología de la endometriosis hay una desregulación en los componentes inmunológicos, como la producción de quimiocinas proinflamatorias, que estimulan la aparición de macrófagos en el tejido endometrial ${ }^{10}$. El incremento de macrófagos activados, así como de la producción de citocinas como el factor de necrosis tumoral alfa (TNF- $\alpha$ ), interleucina (IL) 6 y factor de crecimiento transformante beta (TGF- $\beta$ ), entre otros; activan a los fibroblastos que se encuentran presentes en la pared peritoneal y que, a su vez, generan una rápida producción de IL-6 e IL-8 $8^{11,12}$.

Los macrófagos activados inducen una disminución en la producción de interferón gamma (IFN- $\gamma$ ), lo que conlleva la disminución de la proliferación de los linfocitos $\mathrm{T}$, así como la actividad supresora y el número de células natural killer (NK, asesinas naturales) en la circulación sanguínea, el fluido peritoneal y los tejidos endometriales ectópicos ${ }^{13-15}$. Las células NK son las células efectoras que generalmente reconocen y destruyen tumores celulares. Estudios in vitro han demostrado que al estimular a los linfocitos $T$ de sangre periférica, la producción de IFN- $\gamma$ disminuye, lo que podría sugerir uno de los mecanismos por los cuales hay proliferación de células endometriales ectópicas ${ }^{8}$.

La producción anormal de las citocinas puede ser un paso importante en la patogénesis de la enfermedad; el microambiente en el fluido peritoneal está dado por citocinas como IL-1, IL-6, IL-8 y TNF- $\alpha$, ya que se han encontrado aumentadas ${ }^{15-17}$. El desequilibrio de la producción de citocinas está asociado a la actividad de los macrófagos $\mathrm{y} / \mathrm{o}$ de los linfocitos $\mathrm{T}_{\text {activados }}{ }^{18}$. La secreción de estas citocinas y factores de crecimiento induce la proliferación, diferenciación y mantenimiento endometrial por medio de mecanismos como la adhesión endometrial ectópica y la angiogénesis. Aunado a lo anterior, la evasión de la lisis promueve la sobrevivencia de las células endometriales ectópicas, ya que pueden modular la expresión de moléculas del complejo principal de histocompatibilidad (MHC, major histocompatibility complex) clase I principalmente; estas moléculas son las encargadas de montar la respuesta inmunitaria, 5,19,20.

\section{Marcadores genéticos involucrados en la endometriosis}

Si bien la etiología de la endometriosis no está bien establecida, hay dos factores de suma relevancia que 
en conjunto contribuyen a su desarrollo: por un lado está el fondo genético y por el otro la respuesta inmunitaria. En diversos estudios se propone que ciertos marcadores genéticos están involucrados en la modulación de moléculas importantes que participan durante la respuesta inmunitaria, lo que podría contribuir a la susceptibilidad y/o desarrollo de la patología, además de que el riesgo es heredable ${ }^{21-24}$.

Entre los marcadores que podrían estar involucrados encontramos los genes del sistema HLA (antígenos leucocitarios humanos), los genes de los KIR (immuglobulin-like receptor) y los de los SNP (polimorfismos de nucleótido único, single neuclotide polymorphisms) en moléculas involucradas en la respuesta inmunitaria como las citocinas ${ }^{25-27}$.

\section{Antígeno leucocitario humano}

Determinadas variantes génicas involucradas en los mecanismos inmunológicos son factores importantes en la predisposición genética debido a que participan y regulan la respuesta inmunitaria. Entre los más importantes están los genes ubicados en el MHC, localizados en el brazo corto del cromosoma 6 (6p) en la banda $6 \mathrm{p} 21.3$. Abarca cerca de 4 megabases $(\mathrm{Mb})$ y se caracteriza por ser altamente poligénico (con mayor densidad génica) y polimórfico (con gran variación alélica). Dentro de esta región se encuentran los genes del HLA, que codifican proteínas de superficie indispensables para montar una respuesta inmunitaria y son los genes con mayor asociación, ya que presentan los epítopos peptídicos a las células T. Los diversos alelos de HLA involucrados en la respuesta inmunitaria y la variedad alélica en las diferentes poblaciones étnicas pueden conferir ventajas contra ciertas enfermedades, donde individuos heterocigotos muestren una gran resistencia al presentar un amplio espectro de péptidos ${ }^{28-30}$.

El MHC está dividido en tres regiones: clase I, clase II y clase III. La clase I se encuentra en la superficie de todas las células nucleadas y tienen restricción al presentar solo a linfocitos T CD8+. Las moléculas de clase I son codificadas por varios locus que se dividen en clase la, conformados por los genes clásicos HLA $A, B$ y $C$ que unen péptidos que presentan al receptor de linfocitos T (TCR, del inglés T Cell Receptor) CD4+; y clase $\mathrm{lb}$, que son genes no clásicos HLA E, F y G, los cuales presentan péptidos solo a un subgrupo de células T. La clase II se encuentra únicamente en la superficie de las células presentadoras de antígeno profesionales, de modo que cuando se asocian a péptidos solo son reconocidas por el TCR. Los genes de clase II son clasificados en las familias HLA DP, DN, DM, DO, DQ y DR. La clase III son genes no HLA, pero contienen genes involucrados con la inmunidad, al codificar componentes de la cascada del complemento, TNF y proteínas de choque térmico, entre otros ${ }^{31,32}$.

Debido a que la expresión de los antígenos de HLA está presente en el ciclo menstrual, la asociación del HLA con el desarrollo de endometriosis ha sido estudiada en diferentes poblaciones, en las cuales se reportan datos no concluyentes con respecto a dicha asociación, debido probablemente a la variabilidad étnica entre las poblaciones estudiadas. En relación con lo anterior, en una muestra de mujeres griegas se observó la presencia de moléculas HLA-DR en las células epiteliales glandulares y células estromales del endometrio ectópico, principalmente en las células glandulares, durante la fase secretora del ciclo menstrual y en lesiones de menor tamaño ${ }^{33}$. Por otro lado, el HLA-G es un antígeno no clásico del MHC clase lb y se conoce por inhibir las células T y NK que median la citólisis $^{34,35}$; se observó la presencia de la proteína HLA-G, así como el incremento de su transcripción en el epitelio glandular de lesiones peritoneales endometriales en mujeres con y sin endometriosis, sugiriendo que su elevada expresión pudiera ser una manera de evadir la respuesta inmunitaria adaptativa antiendometrial ${ }^{36}$.

En mujeres asiáticas, la incidencia del alelo HLADQB1*0301 y del alelo HLA-DRB1*1403 fue significativamente mayor en pacientes japonesas con endometriosis comparado con el grupo contro| ${ }^{37,38}$. Otro estudio en mujeres japonesas encontró una asociación significativa entre endometriosis y los alelos HLA-B7 y HLACw*0702; además, se observó una frecuencia aumentada del haplotipo HLA-A24/B*0702/ $\mathrm{CW}^{\star} 0702 / \mathrm{DRB} 1^{*} 0101$ en pacientes con endometriosis comparado con el grupo control ${ }^{39}$. Resultados similares fueron observados en un grupo de mujeres chinas diagnosticadas con endometriosis donde el alelo HLADRB ${ }^{*} 15$ fue significativamente mayor que las mujeres que no padecían esta patología ${ }^{40}$.

\section{Immuglobulin-like receptor}

Las células NK son un componente importante de la respuesta innata, y es la puerta de entrada para la inmunidad adaptativa; entre los receptores que se han 
determinado en la superficie celular de las NK están los KIR, los LIR (receptores leucocitarios tipo inmunoglobulina [lg], del inglés leukocyte lg-like receptor), los miembros de familia de Ly49s y la familia de los receptores CD94/NKG2D ${ }^{41-43}$.

Los receptores KIR son glucoproteínas transmembranales tipo I de la superfamilia de las Ig con dos o tres dominios similares a lg tipo $\mathrm{C} 2$, que se designan KIR2D y KIR3D respectivamente; en general los receptores KIR2D reconocen como ligandos a HLA-C, mientras que los receptores KIR3D reconocen moléculas tipo HLA-A y HLA-B ${ }^{42,43}$.

A los receptores de KIR que contienen dos dominios ITIM (motivo de inhibición del inmunoreceptor basado en tirosina, del inglés immunoreceptor tyrosine-based inhibitory motifs) se les conoce como «L", ya que en su estructura transmembranal tienen dominios ricos en argininas y lisinas, lo que les confiere una actividad inhibitoria, a excepción de KIR2L4, que solo posee un dominio rico en arginina y un solo ITIM. Por lo que los antígenos HLA clase la pueden enviar una señal inhibitoria a las células NK al unirse a los receptores tipo Ig de las células $\mathrm{NK}^{43,44}$.

Mientras que los receptores sin ITIM, pero con ITAM (motivo de activación de inmunoreceptor basado en tirosina, del inglés immunoreceptor tyrosine-based activation motif), conocidos como «S" son activadores. Los dominios extracelulares de las Ig son llamados D0 debido a que es el dominio más distal de la membrana, seguidos de D1 y D2, que es el dominio más cercano a la membrana. El receptor KIR3D contiene los dominios D0, D1 y D2, KIR2DL4 y 2DL5 contienen D0 y D1 y, finalmente, KIR2D contiene D1 y D2 ${ }^{43,44}$.

Los locus de los KIR están codificados en el cromosoma 19q13.4 en una región conocida como complejo de receptores de leucocitos (LRC, del inglés leukocyte receptor complex). Esta familia es inusualmente polimórfica, lo que se observa en una diversa cantidad de haplotipos que se distinguen por una cantidad y clases de genes y alelos de los receptores de $\mathrm{KIR}^{45}$.

Cada haplotipo de KIR está conformado por módulos centroméricos -cen- (3DL3, 2SD2, 2DL2/2DL5B, 3DS3, $3 D P 1,2 D L 1,3 D P 1,2 D L 3)$ y teloméricos -tel- (2DL4, $3 D L 1 / 2 D L 5 A, 2 D S / 2 D S 5,2 D S 1 / 2 D S 4,3 D L 2,3 D S 1)$. Estos módulos están separados de una zona de gran recombinación y de alto grado de polimorfismo (hotspot), la única región repetida es río arriba del gen $K I R 2 D L 4{ }^{45,46}$.

El haplotipo "A» posee cuatro genes KIR inhibitorios (KIR2DL1, KIR2DL3, KIR3DL1 y KIR3DL2) y un gen KIR activador (KIR2DS4), que se unen a sus cuatro ligandos específicos de HLA clase I para los genes inhibitorios (C2 [solo reconocen residuos de aminoácidos de lisina en la posición 80], C1 [solo reconocen residuos de aminoácidos de asparagina en la posición 80], Bw4 y A3/A11) y los alotipos HLA A, C y F para los activadores, respectivamente. Este haplotipo presenta una alta frecuencia génica del alelo KIR2DS4 y su variante polimórfica KIR2DS4v. Los individuos homocigotos para el haplotipo A/A presentan hasta 32 posibles combinaciones génicas de los alelos de $K I R^{43,46}$.

Todos aquellos genes que no estén contenidos en los haplotipos "A» son los que conforman a los haplotipos «B», estos haplotipos consisten en la combinación de cenA/telB, cenB/telA y cenB/telB. Se pueden conformar hasta doce haplotipos que generan hasta 78 diferentes genotipos ${ }^{46,47}$.

La presencia y/o ausencia de los polimorfismos en los genes KIR conllevan una gran variación alélica, especialmente en el haplotipo $A$; dicha variación influencia la especificidad, las vías de señalización, los niveles de expresión y la frecuencia de expresión de los genes $K I R^{43,46,47}$. Dadas estas características, estos alelos en los genes KIR se asocian a diferentes enfermedades ${ }^{48}$. Ahora bien, estos alelos y haplotipos se han estudiado en diversas poblaciones, asociándose con el desarrollo de endometriosis. En un estudio realizado en la población japonesa, demostraron que el haplotipo KIR3DL1+/3DS1-/HLA-Bw4+ está asociado al desarrollo de endometriosis ${ }^{49}$, mientras que en la población polaca se determinó que la presencia de KIR2DS5/ $H L A-C 2$ reduce el riesgo de la enfermedad. En pacientes con endometriosis los alelos KIR2DS5 o la deleción de KIR2DS4 (KIR2DS4del) disminuyen el riesgo de que las lesiones se ubiquen en el peritoneo; por otro lado, KIR2DS4del está asociado a la severidad de la endometriosis ${ }^{50}$.

En mujeres con endometriosis existe una mayor expresión de genes KIR con una actividad inhibitoria, además los haplotipos KIR3DL1/HLA-Bw4 y KIR2DL1/ HLA-C2 están asociados con el desarrollo de los estadios avanzados de la endometriosis ${ }^{51-53}$.

\section{Polimorfismos de nucleótido simple en genes que codifican para citocinas}

Las citocinas y los factores de crecimiento son glucoproteínas, actúan como mediadores clave de la comunicación intracelular del sistema inmunitario y 
pueden actuar en una gran variedad de células ejerciendo efectos proliferativos, citostáticos, quimioatrayentes o de diferenciación celular ${ }^{54}$.

En los genes que codifican para distintas citocinas, se encuentran ciertos marcadores genéticos, los SNP, los cuales pueden modificar la producción y/o su función durante la respuesta inmunitaria, por lo que pueden contribuir a la progresión o resolución entre los diferentes estadios de la endometriosis ${ }^{55,56}$. Los SNP representan hasta el $90 \%$ de las variaciones en el genoma humano, poseen dos alelos e implican el cambio de un par de base por otro, los cuales pueden ser transiciones (purina por purina y viceversa) o translocaciones (purina por pirimídica) que pueden generar cambios sinónimos o no sinónimos ${ }^{57}$.

Algunos de los SNP ocurren en diferentes citocinas asociadas a la respuesta inmunitaria y/o desarrollo de la endometriosis, entre las cuales encontramos las que se explican a continuación.

\section{INTERLEUCINA 1 BETA}

La IL-1 $\beta$ es generada por macrófagos activados y es el primer mediador de las respuestas proinflamatorias $^{58}$. Esta proteína puede aumentar la producción de otras citocinas como la IL-6 y la IL-8, afecta la actividad de los linfocitos $T$, e induce la síntesis de prostaglandinas y la proliferación de fibroblastos estimulados ${ }^{59-61}$.

La IL-1 $\beta$ ha sido aislada en el fluido peritoneal de mujeres con endometriosis ${ }^{62}$ y se ha observado un incremento en los niveles de la expresión de ARNm (ARN mensajero) en macrófagos peritoneales de pacientes en estadios tempranos del padecimiento y de $\mathrm{IL}-1 \beta$ en estadios más severos ${ }^{63}$. Se ha sugerido que la IL-1 $\beta$ promueve la angiogénesis en células endometriales del estroma ${ }^{64}$. También se ha propuesto que interfiere con la vigilancia inmunitaria peritoneal, evitando que sea eliminado en la cavidad peritoneal y que facilite los mecanismos de adhesión en este ambiente $\mathrm{e}^{19,65}$. De tal manera, la expresión anormal de IL-1 $\beta$ puede contribuir al desarrollo de endometriosis.

El gen $I L-1$, localizado en la región proximal del cromosoma $2 q 12$, codifica para las proteínas IL-1 $\alpha$, IL-1 $\beta$ y el antagonista del receptor de IL-1 (IL-1RA) ${ }^{6}$. La IL-1 $\alpha$ y la IL-1 $\beta$ se unen a los mismos receptores y tienen actividades biológicas similares, ambas con un fuerte efecto proinflamatorio ${ }^{54}$.

En el gen IL-1b se encuentran dos polimorfismos, uno localizado en la posición -511 T/C en la región promotora (rs16944) y el segundo en +3953 en el exón 5, donde hay una transición de T por C (rs1143634); estos SNP están relacionados con la producción de IL-1 $\beta$ y con el desarrollo de desórdenes inflamatorios crónicos como enfermedades autoinmunes, cáncer y enfermedades cardiovasculares ${ }^{67-69}$.

El SNP -511 T/C se estudió en la enfermedad gastrointestinal en pacientes húngaros, observándose que los portadores del alelo $T$ producen mayores cantidades de IL-1 $\beta$ que los portadores del alelo $\mathrm{C}^{70}$. Sin embargo, en mujeres asiáticas con endometriosis no se encontraron diferencias estadísticamente significativas en las distribuciones genotípicas ni en las frecuencias alélicas que pudieran relacionar este polimorfismo con la susceptibilidad a adquirir endometriosis ${ }^{55}$.

El SNP +3953 T/C fue analizado en mujeres europeas con endometriosis, en las cuales no se encontró asociación entre este polimorfismo con endometriosis ni con el aumento de la citocina IL-1 $1 \beta^{71}$, mientras que Attar, et al. ${ }^{72}$ encontraron que la frecuencia genotípica TT y la frecuencia alélica $T$ fue mayor en pacientes turcas con endometriosis y que el genotipo TT estuvo asociado a estados avanzados de endometriosis. El alelo $\mathrm{T}$ tiene resultados controvertidos respeto a la producción de IL-1 $\beta$, ya que Santtila, et al. ${ }^{73}$ reportaron una producción menor, mientras que Pociot, et al. ${ }^{74}$ observaron lo contrario, así como el genotipo TT se observó asociado a un aumento en la producción de dicha citocina ${ }^{75}$.

\section{INTERLEUCINA 6}

La IL-6 es una citocina liberada por macrófagos, monocitos, linfocitos $B$ y $T$, fibroblastos, queratinocitos y células endoteliales. Esta citocina regula la inflamación, contribuye a la modulación de la secreción de varias citocinas, es responsable de la maduración celular, promueve la activación de linfocitos $T$ y la diferenciación de linfocitos $B^{54,76}$. La IL-6 está regulada por los niveles de expresión de hormonas, citocinas y factores de transcripción; entre ellos, la IL-1 y el TNF- $\alpha$, que la activan ${ }^{77}$. De modo que, en el fluido peritoneal, esta citocina es secretada por macrófagos en respuesta a varias sustancias como la IL-1 que se encuentra en dicho ambiente ${ }^{78}$.

Se han identificado varias funciones de la IL-6 en el desarrollo de la endometriosis. Por ejemplo, la promueve la proliferación celular del endometrio en condiciones normales y se encuentra en las células 
estromales del endometrio ectópico con la función de inhibir su proliferación $n^{60,79}$. Esto se ha podido comprobar con los niveles elevados de IL-6 en el fluido peritoneal y en circulación sanguínea, y en el incremento de la expresión de IL-6 ARNm que puede estar asociada a la neovascularización presente en la endometriosis ${ }^{60,80-83}$. Además, los niveles de IL-6 en fluido peritoneal se han correlacionado con el grado y el estadio de la enfermedad ${ }^{84,85}$.

El gen IL-6 está localizado en el cromosoma 7p21 y consta de cinco exones y cuatro intrones ${ }^{86}$. Dentro de la región promotora se encuentran los polimorfismos -634 C/G (anteriormente -572 G/C, rs1800796) y -174G/ C (rs1800795). El alelo $C$ en el polimorfismo -634 C/G ha mostrado aumentar la actividad del promotor en respuesta a las citocinas IL-1 $1 \beta$ y TNF- $\alpha^{87,88}$. Mientras que los tres genotipos (GG, GC, CC) del SNP -174 afectan la transcripción de la citocina, el genotipo C/C (de menor incidencia) y los haplotipos que contienen el alelo $\mathrm{C}$ disminuyen los niveles de expresión de IL-6 y se han asociado con menores niveles de IL-6 circulante en personas sanas ${ }^{89,90}$. Por otro lado, se ha relacionado el alelo G del SNP -174 con una mayor transcripción del gen y mayor producción de IL-6 $6^{88}$.

Se ha estudiado la relación de los polimorfismos -634 C/G y -174 G/C con el riesgo de desarrollar endometriosis en diferentes poblaciones, sin embargo, la mayoría no ha encontrado asociaciones que aumenten el riesgo de adquirir la enfermedad; por ejemplo, el polimorfismo -634 C/G en mujeres brasileñas y coreanas $^{91,92}$, y el polimorfismo $-174 \mathrm{G} / \mathrm{C}$ en mujeres europeas, hindúes y coreanas ${ }^{93-95}$. En contraste, el SNP -174 G/C tuvo una asociación significativa con endometriosis en mujeres asiáticas, denotando que este polimorfismo podría ser un factor de riesgo para la susceptibilidad de la endometriosis en esta población ${ }^{96}$.

\section{INTERLEUCINA 8}

La IL-8 es una citocina proinflamatoria que induce la quimiotaxis en neutrófilos, tiene actividad angiogénica local y estimula la proliferación celular ${ }^{97}$. La inflamación y neovascularización observada en y alrededor de los implantes endometriales ectópicos, y la presencia de neutrófilos en estas lesiones es compatible con las funciones biológicas de la IL- $8^{98}$. Además, la adherencia de células endometriales induce la expresión de la IL-8, sugiriendo que esta citocina podría actuar como un factor de crecimiento en la patogénesis de la endometriosis ${ }^{99-101}$.

La IL-8 tiene un efecto directo en la proliferación de las células endometriales del estroma ${ }^{102,103}$. In vivo, células endometriales ectópicas expresan altas concentraciones de IL-8 independientemente de la fase del ciclo menstrual ${ }^{104}$. Además, se ha encontrado en el líquido peritoneal de mujeres con endometriosis y en el líquido quístico del endometrioma está elevado comparado con el líquido de tumores ováricos benignos 20,61,83,105.

El gen IL-8 está localizado en el cromosoma 4q13-q21, está compuesto por cuatro exones, tres intrones y una región promotora ${ }^{106}$. Contiene dos SNPs comunes, uno en la región promotora en las posiciones $-251 \mathrm{~A} / \mathrm{T}$ (rs4073) y el segundo en la posición +781 C/T (rs2227306). El alelo -251A está asociado con niveles elevados de IL-8 e infiltración severa de neutrófilos, que a su vez secretan TNF- $\alpha$, IFN- $\gamma$ e IL-1 $1 \beta^{107}$.

Hasta la fecha ningún estudio ha reportado investigar la asociación de este polimorfismo con endometriosis, sin embargo, se han reportado asociaciones de los polimorfismos con enfermedades infecciosas y cáncer, sobre todo se ha propuesto como biomarcador en el cáncer de ovario ${ }^{108-111}$.

Esto último es relevante, debido a que se ha propuesto una relación entre el desarrollo de endometriosis y tumores malignos de ovario ${ }^{112}$.

Una de las observaciones que asocian estas dos entidades son los perfiles de citocinas en suero y líquido quístico de mujeres con endometriomas, ya que son similares a los que se encuentran en pacientes con tumores quísticos malignos, por ejemplo, los niveles séricos de IL-6 y TNF- $\alpha$ se encuentran elevados en ambos $\operatorname{casos}^{83}$.

\section{INTERLEUCINA 10}

A diferencia de la IL-1 $\beta$, la IL-6 y la IL-8, la IL-10 es una citocina inmunorreguladora, ya que tiene funciones en la respuesta inmunitaria humoral y celular. La IL-10 es producida por macrófagos, monocitos, células $T$, células $B$, células dendríticas, células mastoides y eosinófilos. Como una citocina derivada de los linfocitos T cooperadores tipo 2 (Th2, del inglés T-helper cell type 2), la IL-10 inhibe la secreción de citocinas producidas por los linfocitos T cooperadores tipo 1 (Th1), como IL-1, IL-6 y TNF, que limita la respuesta inflamatoria y regula la diferenciación y proliferación de varias células inmunitarias como linfocitos $T$, linfocitos $B, N K$, 
células presentadoras de antígeno y mastocitos ${ }^{113-115}$. La IL-10 regula la diferenciación y proliferación de los linfocitos $T$ al inhibir a la IL-2 y al IFN- $\gamma$, que son liberados de clones activados de células $\mathrm{T}^{116}$.

La producción elevada de IL-10 en el fluido peritoneal en las mujeres con endometriosis puede sugerir un rol importante en la patogénesis por regulación negativa de la respuesta inmunitaria celular peritoneal y la secreción de citocinas provenientes de células derivadas de Th1 e interfiere con la respuesta proinflamatoria peritoneal, por ejemplo, reduce la activación peritoneal de células $T^{117-119}$.

El gen IL-10 ha sido identificado en el cromosoma 1q3132 y se han descrito tres polimorfismos bialélicos en la región promotora del gen en las posiciones $-1082 \mathrm{G} / \mathrm{A}$ (rs1800896), $-819 \mathrm{C} / \mathrm{T}$ (rs1800871) y -592 C/A (rs1800872) del sitio de inicio de transcripción. Estudios in vitro en células mononucleares de sangre periférica sugieren que los genotipos -1082 G/G, -819 C/C y -592 C/C están asociados con mayor producción de IL-10 $0^{120-122}$.

La asociación de estos SNP solo ha sido estudiada en la población asiática. Por ejemplo, Zhang, et al. ${ }^{123}$ encontraron que la frecuencia del alelo $\mathrm{C}$ en $-819 \mathrm{C} / \mathrm{T}$ o en $-592 \mathrm{C} / \mathrm{A}$ es significativamente mayor en pacientes asiáticas con endometriosis, por lo que podrían considerarse alelos de susceptibilidad. Sin embargo, un metaanálisis realizado por Fan, et al. ${ }^{124}$ solo observó significancia en la asociación del alelo $C$ en el SNP $-592 \mathrm{C} / \mathrm{A}$ y no en los otros 2 polimorfismos (-1082 G/A y $-819 \mathrm{C} / \mathrm{T})$.

Aunado a esto, Zhang, et al. ${ }^{123}$ observaron que la frecuencia genotípica CC y el alelo C en -819 C/T o $-592 \mathrm{C} / \mathrm{A}$, así como el haplotipo ACC de los tres SNP de IL-10 se encontraron incrementados estadísticamente en pacientes con endometriosis moderada y severa. Por el contrario, Fan, et al. ${ }^{124}$ no encontraron ninguna asociación entre la severidad de la enfermedad y los polimorfismos de IL-10. Esto sugiere que los polimorfismos $-819 \mathrm{C} / \mathrm{T}$ y $-592 \mathrm{C} / \mathrm{A}$ de $\mathrm{L}$ L-10 tienen una función importante en la endometriosis, al conferir susceptibilidad de desarrollarla, y probablemente pudieran estar involucrados en el desarrollo de los diferentes estadios.

\section{Factor de necrosis tumoral alfa}

El TNF- $\alpha$ es un inmunomodulador y una citocina proinflamatoria producida por neutrófilos, linfocitos activados, macrófagos y NK. Entre sus funciones se encuentra la activación de factores de crecimiento, citocinas y quimioatrayentes; además, afecta la síntesis y estimulación de las moléculas de adhesión ${ }^{125,126}$.

En mujeres con endometriosis se han encontrado niveles elevados en el líquido peritoneal y correlacionan con el estadio de la enfermedad ${ }^{127}$. Además, se encontró que los niveles séricos de TNF- $\alpha$ fueron mayores en mujeres con endometriomas que en mujeres con tumores ováricos benignos, pero estos niveles fueron similares a los observados en tumores malignos ováricos ${ }^{83}$.

Se ha sugerido que los niveles elevados de TNF- $\alpha$ facilitan la implantación peritoneal del endometrio ectópico, ya que in vitro se observó un aumento significativo en la adherencia de las células endometriales del estroma a las células mesoteliales tratadas previamente con TNF- $\alpha^{128}$. El gen TNF- $\alpha$ se encuentra en el cromosoma $6 \mathrm{p} 21$, donde se encuentran los siguientes polimorfismos en la región promotora: -238 G/A (rs361525), -308 G/A (rs3091256) y -1031 T/C (rs1799964). El alelo $A$ en el SNP -308 es raro (TNF2), pertenece al haplotipo HLA-A1-B8-DR3-DQ2 y se asocia con mayor producción de TNF- $\alpha^{129,130}$. La producción de TNF- $\alpha$ se ha visto afectada por el SNP $-1031 \mathrm{~T} / \mathrm{C}^{95}$.

Un metaanálisis en mujeres asiáticas con endometriosis encontró que el SNP -238 A/G tiene una significativa asociación con dicha enfermedad, por lo que este polimorfismo es un factor de riesgo para adquirir endometriosis. Por el contrario, el SNP -1031 T/C en endometriosis está asociado con el alelo $\mathrm{T}$, sugiriendo que el polimorfismo podría reducir el riesgo de adquirir la enfermedad. Finalmente, no se encontraron asociaciones con el SNP $-308 \mathrm{~A} / \mathrm{G}^{114}$.

\section{Factor de crecimiento transformador beta}

EI TGF- $\beta$ es una familia de citocinas que inhibe o estimula el crecimiento celular, está involucrado en la proliferación y diferenciación celular, sintetiza proteínas de la matriz extracelular y participa en la estimulación de la angiogénesis, la fibrosis y las respuestas inmunitarias ${ }^{131,132}$. El TGF- $\beta$ inhibe la actividad de las células NK e induce angiogénesis y proliferación de las células endometriales del estroma ${ }^{133}$.

Su presencia se ha observado en implantes endometriales en ratas a las que se les indujo endometriosis quirúrgicamente y se ha registrado que sus niveles están aumentados en mujeres con endometriosis $y$ en mujeres con adhesiones pélvicas en 
comparación con una pelvis normal ${ }^{134}$. Este aumento puede ser debido a una respuesta pélvica inflamatoria secundaria a la presencia de sangre o endometrio que se desprende con la menstruación en la pelvis ${ }^{135}$. El tratamiento in vitro de las células endometriales del estroma con TGF- $\beta$ puede desencadenar la apoptosis de las células inmunitarias activadas, permitiendo que las células endometriales en el líquido peritoneal escapen a la vigilancia inmunitaria, se implanten y crezcan ${ }^{136}$.

El gen TGF-b está localizado en el cromosoma 19q13.1, donde el polimorfismo -509 C/T (rs1800469) está asociado al desarrollo de endometriosis, tanto el alelo como y el genotipo TT están asociados con el incremento en la producción de TGF- $\beta 1$, así como con una mayor susceptibilidad a desarrollar endometriosis en mujeres asiáticas ${ }^{55}$. Aunado a lo anterior, está asociado con una actividad inhibitoria por TGF-1 tanto de las respuestas tipo Th1 y Th2 ${ }^{137}$. De tal forma, que esta citocina y su receptor tienen un papel importante en el desarrollo y cronicidad de la enfermedad ${ }^{133}$.

\section{Conclusión}

Si bien cada una de las teorías planteadas explican el posible mecanismo de la presencia de tejido endometrial ectópico, se sabe que en mujeres sanas dicha enfermedad no podría desarrollarse, por lo que la etiología inmunitaria y el desarrollo de un proceso proinflamatorio se ha sustentado como la más viable, debido a que hay una inadecuada inmunovigilancia con el consecuente desarrollo, progresión y persistencia de endometriosis. Aunado a esto, el fondo genético es un factor de suma importancia, ya que ciertos marcadores genéticos están asociados a moléculas que participan y regulan la respuesta inmunitaria, por lo tanto puede influir en su modulación. Comprender cómo el fondo genético influye en la modulación de la respuesta inmunitaria y su consecuente participación en el desarrollo de la endometriosis contribuirá a un mejor manejo y tratamiento de las pacientes.

\section{Financiamiento}

Este protocolo fue financiado por el Programa Presupuestal E015-2018 de Investigación y Desarrollo Tecnológico en Salud, otorgado por el Instituto de Seguridad y Servicios Sociales de los Trabajadores del Estado.

\section{Conflicto de intereses}

Los autores declaran no tener conflictos de intereses.

\section{Responsabilidades éticas}

Protección de personas y animales. Los autores declaran que para esta investigación no se han realizado experimentos en seres humanos ni en animales.

Confidencialidad de los datos. Los autores declaran que han seguido los protocolos de su centro de trabajo sobre la publicación de datos de pacientes.

Derecho a la privacidad y consentimiento informado. Los autores han obtenido el consentimiento informado de los pacientes y/o sujetos referidos en el artículo.

\section{Bibliografía}

1. Hickey M, Ballard K, Farquhar C. Endometriosis. BMJ. 2014:348:1-9.

2. Ballard KD, Seaman HE, de Vries CS, Wright JT. Can symptomatology help in the diagnosis of endometriosis? Findings from a national case-control study-part 1. BJOG. 2008;15(11):1382-91.

3. López Monsalvo A, Adame Pinacho R. Endometriosis. Perinatol Reprod Hum. 2006;21(4).

4. Hsu AL, Khachikyan I, Stratton P. Invasive and non-invasive methods for the diagnosis of endometriosis. Clin Obstet Gynecol. 2010;53(2): 413-9.

5. Sourial S, Tempest N, Hapangama DK. Theories on the pathogenesis of endometriosis. Int J Reprod Med. 2014;2014:179515.

6. Ahn S, Monsanto S, Miller C, Singh S, Thomas R, Tayade CH. Pathophysiology and immune dysfunction in endometriosis. BioMed Res Int. 2015;2015:795976.

7. Sampson JA. Metastatic or embolic endometriosis, due to the menstrual dissemination of endometrial tissue into the venous circulation. Am J Pathol. 1927:3(2):93-110.

8. Szyllo K, Tchorzewski H, Banasik M, Glowacka E, Lewkowicz P, Kamer-Bartosinska $A$. The involvement of $T$ lymphocytes in the pathogenesis of endometriotic tissues overgrowth in women with endometriosis. Mediators Inflamm. 2003;12(3):131-8.

9. Dmowski WP, Steele RW, Baker GF. Deficient cellular immunity in endometriosis. Am J Obstet Gynecol. 1981;141:377-83.

10. Hornung D, Klingel K, Dohrn K, Kandolf R, Wallwiener D, Taylor RN. Regulated on activation, normal T-cell-expressed and secreted mRNA expression in normal endometrium and endometriotic implants: assessment of autocrine/paracrine regulation by in situ hybridization. Am J Pathol. 2001;158(6):1949-54.

11. D'Hooghe TM, Xiao L, Hill JA. Cytokine profiles in autologous peritoneal fluid and peripheral blood of women with deep and superficial endometriosis. Arch Gynecol Obstet. 2001;265(1):40-4.

12. Buckley CD, Pilling D, Lord JM, Akbar AN, Scheel-Toellener, Salmon M. Fibroblasts regulate the switch from acute resolving to chronic persistent inflammation. Trends Immunol. 2001;22:199-204.

13. Oosterlynck DJ, Meuleman CR, Waer MR, Konickx PR, Vandeputte M. Immunosuppressive activity of peritoneal fluid in women with endometriosis. Obstet Gynecol. 1993;82:206-12.

14. Ho HN, Wu MY, Chao KH, Chen CD, Chen SU, Chen HF, et al. Decrease in interferon gamma production and impairment of T-lymphocyte proliferation in peritoneal fluid of women with endometriosis. Am J Obstet Gynecol. 1996;175:1236-41.

15. Ho HN, Wu MY, Yang YS. Peritoneal cellular immunity and endometriosis. Amer J Reprod Immunol. 1997;38:400-12.

16. Zhang X, Xu H, Lin J, Qian Y, Deng L. Peritoneal fluid concentrations of interleukin-17 correlate with the severity of endometriosis and infertility of this disorder. BJOG. 2005;112:1153-5.

17. Calhaz-Jorge C, Costa AP, Santos MC, Palma-Carlos ML. Peritoneal fluid concentrations of interleukin-8 in patients with endometriosis de- 
pend on the severity of the disorder and are higher in the luteal phase. Hum Reprod. 2003;18;593-7.

18. D'Hooghe TM, Bambra CS, Xiao L, Peixe K, Hill JA. Effect of menstruation and intrapelvic injection of endometrium on inflammatory parameters of peritoneal fluid in the baboon (Papio Anubis and Papio cynocephalus). Am J Obstet Gynecol. 2001:184(5):917-25.

19. Sillem M, Prifti S, Monga B, Arslic T, Runnebaum B. Integrin-mediated adhesion of uterine endometrial cells from endometriosis patients to extraceIlular matrix proteins is enhanced by tumor necrosis factor alpha (TNF alpha) and interleukin-1 (IL-1). Eur J Obstet Gynecol Reprod Biol. 1999;87(2):123-7.

20. Fasciani A, D’Ambrogio G, Bocci G, Monti M, Genazzani AR, Artini PG. High concentrations of the vascular endothelial growth factor and interleukin-8 in ovarian endometriomata. Mol Hum Reprod. 2000;6(1):50-4.

21. Hadfield RM, Mardon HJ, Barlow DH, Kennedy SH. Endometriosis in monozygotic twins. Fertil Steril. 1997;68(5):941-2.

22. Kennedy S. The genetics of endometriosis. Eur J Obstet Gynecol Reprod Biol. 1999;82(2):129-33.

23. Zondervan KT, Cardon LR, Kennedy SH. The genetic basis of endometriosis. Curr Opin Obstet Gynecol. 2001;13:309-14.

24. Stefansson $H$, Geirsson RT, Steinthorsdottir V, Jonsson $H$, Manolescu A, Kong $A$, et al. Genetic factors contribute to the risk of developing endometriosis. Hum Reprod. 2002;17(3):555-9.

25. Falconer H, D'Hooghe TM, Fried G. Endometriosis and genetic polymorphisms. Obstet Gynecol Surv. 2007;62:616-28.

26. Montgomery GW, Nyholt DR, Zhao ZZ, Treloar SA, Painter JN, Missmer $\mathrm{SA}$, et al. The search for genes contributing to endometriosis risk. Hum Reprod Update. 2008;14(5):447-57.

27. Rahmioglu N, Missmer SA, Montgomery GW, Zondervan KT. Insights into assessing the genetics of endometriosis. Curr Obstet Gynecol Rep. 2012;1:124-37.

28. Traherne JA. Human MHC architecture and evolution: implications for disease association studies. Int J Immunogenet. 2008;35:179-92.

29. Knapp LA. The ABCs of MHC. Evol Anthr. 2005;14:28-37.

30. Horton R, Wilming L, Lovering R, Bruford EA, Khodiyar VK, Lush MJ, et al. Gene map of the extended human MHC. Nat Rev Genet: 2004;5:889-99.

31. Shankarkumar U. The Human Leukocyte Antigen (HLA) System. Int J Hum Genet. 2004;4(2):91-103.

32. Granados Arriola J, Salgado Galicia N, Ortega-Hernández E, Mena L, Soler M \& Escamilla Tilch M. Inmunología: apuntes para estudiantes de biomedicina. $1^{\circ}$ ed. México: Ed. Prado S.A. de C.V.; 2018.

33. Koumantakis E, Panayiotides J, Goumenou A, Ziogos ECh, Margariti A Kalapothaki V, et al. Different HLA-DR expression in endometriotic and adenomyotic lesions: correlation with transvaginal ultrasonography findings. Arch Gynecol Obstet. 2010;281(5):851-6.

34. Hunt JS, Petroff MG, Mclntire RH, Ober C. HLA-G and immune tolerance in pregnancy. FASEB J. 2005;19(7):681-93.

35. Le Gal FA, Riteau B, Sedlik C, Khalil-Daher I, Menier C, Dausset J, et al. HLA-G-mediated inhibition of antigen-specific cytotoxic T lymphocytes. Int Immunol. 1999:11(8):1351-6.

36. Barrier BF, Kendall BS, Ryan CE, Sharpe-Timms KL. HLA-G is expressed by the glandular epithelium of peritoneal endometriosis but not in eutopic endometrium. Hum Reprod. 2006;21(4):864-9.

37. Ishii K, Takakuwa K, Kashima K, Tamura M, Tanaka K. Associations between patients with endometriosis and HLA class II; the analysis of HLADQB1 and HLADPB1 genotypes. Hum Reprod. 2003;18(5):985-9.

38. Ishii K, Takakuwa K, Mitsui T, Tanaka K. Human studies on the human leukocyte antigen-DR in patients with endometriosis: genotyping of HLADRB1 alleles. Reproduction. 2002;17(3):560-3.

39. Kitawaki J, Obayashi H, Ohta M, Kado N, Ishihara H, Koshiba H, et al. Genetic contribution of the interleukin-10 promoter polymorphism in endometriosis susceptibility. Am J Reprod Immunol. 2002;47:12-8.

40. Wang X, Liu C, Lin Q, Fang X, Lin L, Mei Q. Study on polymorphism of human leukocyte antigen-DRB1 allele in patients with endometriosis. Chung Hua Fu Chan Ko Tsa Chih. 2002;37:346-8.

41. Norman PJ, Parham P. Complex interactions: The immunogenetics of human leukocyte antigen and killer cell immunoglobulin-like receptors. Semin Hematol. 2005:4:62-75.

42. Sawicki M, Dimasi N, Natarajan K, Wang J, Margulies DH, Mariuzza RA. Structural basis of $\mathrm{MHC}$ class I recognition by natural killer cell receptors. Immunol Rev. 2001;181:52-65.

43. Deng L, Mariuzza RA. Structural basis for recognition of MHC and MHC-like ligands by natural killer cell receptor. Semin Immunol. 2006;18(3):159-66.

44. Hsu K, Chida S, Geraghty D, Dupont B. The killer cell immunoglobulin-like receptor (KIR) genomic region: gene-order, haplotypes and allelic polymorphisms. Immunol Rev. 2002;190:40-52.

45. Trowsdale J, Barten R, Haude A, Stewart CA, Beck S, Wilson MJ. The genomic context of natural killer receptor extended gene families. Immunol Rev. 2001;181:20-38.
46. Manser A, Weinhold S, Uhrberg M. Human KIR repertories: shaped by genetic diversity and evolution. Immunol Rev. 2015;267:178-96.

47. Bashirova AA, Martin MP, McVicar DW, Carrington M. The killer immunoglobulin-like receptor gene cluster: tuning the genome for defense. Annu Rev Genomics Hum Genet. 2006;7:277-300.

48. Ivarsson MA, Michaëlsson J, Fauriat $\mathrm{C}$. Activating killer cell Ig-like receptors in health and disease. Front Immunol. 2014;5(184):1-9.

49. Kitawaki J, Xu B, Ishihara H, Fukui M, Hasegawa G, Nakamura N, et al. Association of killer cell immunoglobulin-like receptor genotypes with susceptibility to endometriosis. Am J Reprod Immunol. 2007;58:481-6.

50. Nowak I, Ploski R, Barez E, Dziunycz P, Kamiðski P, Kostrzewa G, et al. KIR2D55 in the presence of HLA-C C2 protects against endometriosis. Immunogenetics. 2015;67(4):203-9.

51. Wilson TJ, Hertzog PJ, Angus D, Munnery L, Wood EC, Kola I. Decreased natural killer cell activity in endometriosis patients: relationship to disease pathogenesis. Fertil Steril. 1994;62(5):1086-8.

52. Wu MY, Yang JH, Chao KH, Hwang JL, Yang YS, Ho HN. Increase in the expression of killer cell inhibitory receptors on peritoneal natural killer cells in women with endometriosis. Fertil Steril. 2000;74(6):1187-91.

53. Matsuoka S, Maeda N, Izumiya C, Yamashita C, Nishimori Y, Fukaya T. Expression of inhibitory-motif killer immunoglobulin-like receptor, KIR2DL1, is increased in natural killer cells from women with pelvic endometriosis. Am J Reprod Immunol. 2005;53(5):249-54.

54. Lebovic DI, Mueller MD, Taylor RN. Immunobiology of endometriosis. Fertil Steril. 2001;75(1):1-10.

55. Hsieh YY, Chang CC, Tsai FJ, Peng CT, Yeh LS, Lin CC. Polymorphism for transforming growth factor beta 1-509 (TGF-b1-509): association with endometriosis. Biochem Genet. 2005;43(5):203-10.

56. Luisi S, Galleri L, Marini F, Ambrosini G, Brandi ML, Petraglia F. Estrogen receptor gene polymorphisms are associated with recurrence of endometriosis. Fertil Steril. 2006;85(3):764-6.

57. Checa Caratachea MA. Polimorfismos genéticos: importancia y aplicaciones. Rev Inst Nal Enf Resp Mex. 2007;20(3):213-21.

58. Dinarello CA. An update on human interleukin-l: from molecular biology to clinical relevance. J Clin Immunol. 1985;5(5):287-90.

59. Rossi V, Breviario F, Ghezzi P, Dejana E, Mantovani A. Prostacyclin synthesis induced by vascular cells by interleukin-1. Science. 1985;229(4709):174-6.

60. Tseng JF, Ryan IP, Milam TD, Murai JT, Schriock ED, Landers DV, et al. Interleukin-6 secretion in vitro is up-regulated in ectopic and eutopic endometrial stromal cells from women with endometriosis. J Clin Endocrinol Metab. 1996;81(3):1118-22.

61. Arici A, Tazuke SI, Attar E, Kliman HJ, Olive DL. Interleukin-8 concentration in peritoneal fluid of patients with endometriosis and modulation of interleukin-8 expression in human mesothelial cells. Mol Hum Reprod. 1996;2:40-5.

62. Hill $\mathrm{J}$, Anderson D. Lymphocyte activity in the presence of peritoneal fluid from fertile women and infertile women with and without endometriosis. Am J Obstet Gynecol. 1989;161:861-4.

63. Raiter-Tenenbaum A, Baranao RI, Etchepareborda JJ, Meresman GF, Rumi LS. Functional and phenotypic alterations in peritoneal macrophages from patients with early and advanced endometriosis. Arch Gynecol Obstet. 1998;261(3):147-57.

64. Lebovic DI, Bentzien F, Chao VA, Garrett EN, Meng YG, Taylor RN. Induction of an angiogenic phenotype in endometriotic stromal cell cultures by interleukin-1. Mol Hum Reprod. 2000;6(3):269-75.

65. Viganò $P$, Gaffuri B, Somigliana E, Busacca M, Di Blasio AM, Vignali M. Expression of intercellular adhesion molecule (ICAM)-1 mRNA and protein is enhanced in endometriosis versus endometrial stromal cells in culture. Mol Hum Reprod. 1998;4(12):1150-6.

66. Copeland NG, Silan CM, Kingsley DM, Jenkins NA, Cannizzaro LA, Croce CM, et al. Chromosomal location of murine and human IL-1 receptor genes. Genomics. 1991;9(1):44-5.

67. Mark LL, Haffajee AD, Socransky SS, Kent RL Jr, Guerrero D, Kornman $\mathrm{K}$, et al. Effect of the interleukin-1 genotype on monocyte IL-1beta expression in subjects with adult periodontitis. J Periodontal Res. 2000;35:172-77.

68. Shen J, Arnett DK, Peacock JM, Parnell LD, Kraja A, Hixson JE, et al. Interleukin $1 \beta$ genetic polymorphisms interact with polyunsaturated fatty acids to modulate risk of the metabolic syndrome. J Natur. 2007;137(8):1846-51.

69. Cañas M, Morán $Y$, Rivero MB, Bohórquez A, Villegas $V$, Rendón $Y$, et al. Polimorfismo genético de interleuquina-1: asociación con cáncer gástrico en la población de alto riesgo del Centroccidente de Venezuela. Rev Med Chil. 2009;137(1):63-70.

70. Nemetz A, Nosti-Escanilla MP, Molnar T, Köpe A, Kovács A, Fehér J, et al. IL1B gene polymorphisms influence the course and severity of inflammatory bowel disease. Immunogenetics. 1999;49:527-31.

71. Wieser F, Fabjani G, Tempfer C, Schneeberger C, Sator M, Huber J, et al. Analysis of an interleukin-6 gene promoter polymorphism in women 
with endometriosis by pyrosequencing. J Soc Gynecol Investig. 2003;10(1):32-6.

72. Attar R, Agachan B, Kucukhuseyin O, Toptas B, Attar E, Isbir T. Association of interleukin 1beta gene $(+3953)$ polymorphism and severity of endometriosis in Turkish women. Mol Biol Rep. 2010;37(1):369-74.

73. Santtila S, Savinainen K, Hurme M. Presence of the IL-1RA allele 2 (IL1RN-2) is associated with enhanced IL-1beta production in vitro. Scand J Immunol. 1998;47:195-8.

74. Pociot F, Molvig J, Wogensen L, Worsaae H, Nerup J. A Taql polymorphism in the human interleukin-1 $\beta$ (IL-1 $\beta$ ) gene correlates with IL-1 $\beta$ secretion in vitro. Eur J Clin Invest. 1992;22(6):396-402.

75. Hall SK, Perregaux DG, Gabel CA, Woodworth T, Durham LK, Huizinga TW, et al. Correlation of polymorphic variation in the promoter region of the interleukin-1 beta gene with secretion of interleukin-1 beta protein. Arthritis Rheum. 2004;50:1976-83.

76. Kishimoto T, Hirano T. Molecular regulation of the B lymphocyte response. Ann Rev Immunol. 1988;6:485-9.

77. Hirano T. Interleukin 6 and its receptor: ten years later. Int Rev Immunol. 1998;16(3-4):249-84.

78. Sironi M, Breviario F, Proserpio P, Biondi A, Vecchi A, Van Damme J, et al. IL-1 stimulates IL-6 production in endothelial. J Immunol. 1989;142(2):549-53.

79. Zarmakoupis PN, Rier SE, Maroulis GB, Becker JL. Inhibition of human endometrial stromal cell proliferation by interleukin 6. Hum Reprod. 1995;10(9):2395-9.

80. Keenan JA, Chen TT, Chadwell NL, Torry DS, Caudle MR. Interferon-gamma (IFN- $\gamma$ ) and interleukin-6 (IL-6) in peritoneal fluid and macrophage-conditioned media of women with endometriosis. Am J Reprod Immunol. 1994;32:180-3.

81. Odukoya OA, Ajjan R, Lim K, Watson PF, Weetman AP, Cooke ID. The pattern of cytokine mRNA expression in ovarian endometriomata. Mol Hum Reprod. 1997:3:393-7.

82. Tsudo T, Harada T, Iwabe T, Tanikawa M, Nagano Y, Ito M, et al. Altered gene expression and secretion of interleukin-6 in stromal cells derived from endometriotic tissues. Fertil Steril. 2000;73(2):205-11.

83. Daraï E, Detchev R, Hugol D, Quang NT. Serum and cyst fluid levels of interleukin (IL) 6, IL8 and tumour necrosis factor-alpha in women with endometriomas and benign and malignant cystic ovarian tumours. Hum Reprod. 2003;18(8): 1681-5.

84. Cheong YC, Shelton JB, Laird SM, Richmond M, Kudesia G, Li TC, et al. IL-1, IL-6 and TNF-alpha concentrations in the peritoneal fluid of women with pelvic adhesions. Hum Reprod. 2002;17(1):69-75.

85. Khan KN, Masuzaki H, Fujishita A. Association of interleukin-6 and estradiol with hepatocyte growth factor in peritoneal fluid of women with endometriosis. Acta Obstet Gynecol Scand. 2002;81:764-71.

86. Yasukawa K, Hirano T, Watanabe Y, Muratani K, Matsuda T, Nakai S, et al. Structure and expression of human B cell stimulatory factor-2 (BSF-2/LL-6) gene. EMBO J. 1987;6(10):2939-45.

87. Brull DJ, Montgomery HE, Sanders J, Dhamrait S, Luong L, Rumley A, et al. Interleukin-6 gene 174g-c and 572g-c promoter polymorphisms are strong predictors of plasma interleukin-6 levels after coronary artery bypass surgery. Arterioscler Thromb Vasc Biol. 2001;21:1458-63.

88. Ferrari LS, Ahn-Luong EL, Garnero LP, Humphries SE, Greenspan SL. Two promoter polymorphisms regulating Interleukin-6 gene expression are associated with circulating levels of C-Reactive Protein and markers of bone resorption in postmenopausal women. J Clin Endocrinol Metab. 2003;88(1):255-9.

89. Fishman D, Faulds G, Jeffery R, Mohamed-Ali V, Yudkin JS, Humphries $\mathrm{S}$, et al. The effect of novel polymorphisms in the interleukin-6 (IL-6) gene on IL-6 transcription and plasma IL-6 levels, and an association with systemic- onset juvenile chronic arthritis. J Clin Invest. 1998;102:1369-76.

90. Terry CF, Loukaci V, Green FR. Cooperative influence of genetic polymorphisms on interleukin 6 transcriptional regulation. J Biol Chem. 2000;275(24):18138-44

91. Bessa N, Francisco D, Andres M, Gueuvoghlanian-Silva BY, Podgaec S, Fridman C. Polymorphisms of ICAM- 1 and IL- 6 genes related to endometriosis in a sample of Brazilian women. J Assist Reprod Genet. 2016;33(11):1487-92

92. Chae SJ, Lee GH, Choi YM, Hong MA, Kim JM, Lee KS, et al. Intercellular adhesion molecule- 1 and interleukin- 6 gene polymorphisms in patients with advanced-stage endometriosis. Gynecol Obstet Invest. 2010;70(1):34-9.

93. Wieser F, Hefler L, Tempfer C, Vlach U, Schneeberger C, Huber J, et al. Polymorphism of the interleukin-1beta gene and endometriosis. J Soc Gynecol Investig. 2003:10(3):172-5.

94. Bhanoori M, Babu KA, Deenadayal M, Kennedy S, Shivaji S. The interleukin-6 -174G/C promoter polymorphism is not associated with endometriosis in South Indian women. J Soc Gynecol Investig. 2005:12(5):365-9.

95. Lee MK, Park AJ, Kim DH. Tumor necrosis factor-alpha and interleukin- 6 promoter gene polymorphisms are not associated with an increased risk of endometriosis. Fertil Steril. 2002;77(6):1304-5.
96. Li J, Chen Y, Wei S, Wu H, Liu C, Huang Q, et al. Tumor necrosis factor and interleukin- 6 gene polymorphisms and endometriosis risk in Asians: a systematic review and meta-analysis. Ann Hum Genet. 2014;78(2): 104-16.

97. Koch AE, Polverini PJ, Kunkel SL, Harlow LA, DiPietro LA, Elner VM, et al. Interleukin-8 is a macrophage-derived mediator of angiogenesis. Science. 1992;258:1798-801.

98. Van Deuren M, Dofferhoff ASM, van der Meer JWM. Cytokines and the response to infection. J Pathol. 1992;168:349-56.

99. Arici A. Local cytokines in endometrial tissue: the role of Interleukin-8 in the pathogenesis of endometriosis. Ann NY Acad Sci. 2002;955(1):101-9.

100. Arici A, Seli E Senturk LM, Gutierrez LS, Oral E Taylor HS, et al. Interleukin-8 in the human endometrium. J Clin Endocrinol Metab. 1998;83(5):1783-87.

101. Harada T, Enatsu A, Mitsunari M, Nagano Y, Ito M, Tsudo T, et al. Role of cytokines in progression of endometriosis. Gynecol Obstet Invest. 1999;47(Suppl 1):34-9.

102. Arici A, Seli E, Zeyneloglu HB, Senturk LM, Oral E, Olive DL. Interleukin-8 induces proliferation of endometrial stromal cells: a potential autocrine growth factor. J Clin Endocrinol Metab. 1998;83(4):1201-5.

103. Iwabe T, Harada T, Tsudo T, Tanikawa M, Onohara Y, Terakawa N. Pathogenetic significance of increased levels of interleukin-8 in peritoneal fluid of patients with endometriosis. Fertil Steril. 1998;69(5):924-30.

104. Akoum A, Lawson C, McColl S, Villeneuve M. Ectopic endometrial cells express high concentrations of interleukin (IL)-8 in vivo regardless of the menstrual cycle phase and respond to oestradiol by up- regulating IL-1-induced IL-8 expression in vitro. Mol Hum Reprod. 2001;7(9): 859-66.

105. Fasciani A, D’Ambrogio G, Bocci G, Luisi S, Artini PG, Genazzani AR. Vascular endothelial growth factor and interleukin-8 in ovarian cystic pathology. Fertil Steril. 2001;75(6):1218-21.

106. Mukaida N, Shiroo M, Matsushima K. Genomic structure of the human monocyte-derived neutrophil chemotactic factor IL-8. J Immunol. 1989:143(4):1366-71.

107. Taguchi A, Ohmiya N, Shirai K, Mabuchi N, Itoh A, Hirooka Y, et al. Interleukin-8 promoter polymorphism increases the risk of atrophic gastritis and gastric cancer in Japan. Cancer Epidemiol Biomarkers Prev. 2005;14(11):2487-93.

108. Hull J, Thomson A, Kwiatkowski D. Association of respiratory syncytial virus bronchiolitis with the interleukin 8 gene region in UK families. Thorax. 2000;55(12):1023-7.

109. Lokshin AE, Winans M, Landsittel D, Marrangoni AM, Velikokhatnaya L, Modugno $\mathrm{F}$, et al. Circulating IL-8 and anti-IL-8 autoantibody in patients with ovarian cancer. Gynecol Oncol. 2006;102(2):244-51.

110. Rafrafi A, Chahed B, Kaabachi S, Kaabachi W, Maalmi H, Hamzaoui K, et al. Association of IL-8 gene polymorphisms with non-small cell lung cancer in Tunisia: A case control study. Hum Immunol. 2013;74(10): |1368-74.

111. Koensgen D, Bruennert D, Ungureanu S, Sofroni D, Braicu El, Sehouli $\mathrm{J}$, et al. Polymorphism of the IL-8 gene and the risk of ovarian cancer. Cytokine. 2015;71(2):334-8.

112. Greene AD, Lang, SA, Kendziorski JA. Endometriosis: where are we and where are we going? Reproduction. 2016;152(3):R63-R78.

113. Gallagher PM, Lowe G, Fitzgerald T, Bella A, Greene CM, McElvaney $\mathrm{NG}$, et al. Association of IL-10 polymorphism with severity of illness in community acquired pneumonia. Thorax. 2003;58(2):154.

114. Li MC, He SH. IL-10 and its related cytokines for treatment of inflammatory bowel disease. World J Gastroenterol. 2004:10(5):620-5

115. Núñez C, Alecsandru D, Varade J, Polanco I, Maluenda C, Fernández-Arquero $\mathrm{M}$, et al. Interleukin-10 haplotypes in celiac disease in the Spanish population. BMC Med Genet. 2006;7:32-6.

116. Ebert EC. IL-10 enhances IL-2-induced proliferation and cytotoxicity by human intestinal lymphocytes. Clin Exp Immunol. 2000;119(3):426-32.

117. Ho HN, Wu MY, Chao KH, Chen CD, Chen SU, Yang YS. Peritoneal interleukin-10 increases with decrease in activated CD4+ T lymphocytes in women with endometriosis. Hum Reprod. 1997;12(11):2528-33.

118. Lee KS, Baek DW, Kim KH, Shin BS, Lee DH, Kim JW, et al. IL-10-dependent down-regulation of $\mathrm{MHC}$ class II expression level on monocytes by peritoneal fluid from endometriosis patients. Int Immunopharmacol. 2005:5(12):1699-712.

119. Agic $A, X u H$, Finas D, Banz C, Diedrich K, Hornung D. Is endometriosis associated with systemic subclinical inflammation? Gynecol Obstet Invest. 2006;62(3):139-47.

120. Eskdale J, Kube D, Tesch H, Gallagher G. Mapping of the human IL10 gene and further characterization of the 5 ' flanking sequence. Immunogenetics. 1997;46(2):120-8.

121. Turner DM, Williams DM, Sankaran D, Lazarus M, Sinnott PJ, Hutchinson IV. An investigation of polymorphism in the interleukin-10 gene promoter. Eur J Immunogenet. 1997:24:1-8. 


\section{A. Cortes-Algara, et al.: Inmunogenética de la endometriosis}

122. Opdal SH. IL-10 gene polymorphisms in infectious disease and SIDS. FEMS Immunol Med Microbiol. 2004;42:48-52.

123. Zhang $X$, Hei $P$, Deng L, Lin J. Interleukin-10 gene promoter polymorphisms and their protein production in peritoneal fluid in patients with endometriosis. Mol Hum Reprod. 2007;13(2):135-40.

124. Fan W, Li S, Chen Q, Huang Z, Ma Q, Xiao Z. Association between interleukin-10 promoter polymorphisms and endometriosis: A meta-analysis. Gene. 2013;515(1):49-55.

125. Azmy IA, Balasubramanian SP, Wilson AG, Stephenson TJ, Cox A Brown NJ, et al. Role of tumour necrosis factor gene polymorphisms $(-308$ and -238$)$ in breast cancer susceptibility and severity. Breast Cancer Res. 2004;6(4):R395-R400.

126. Sharma S, Rathored J, Ghosh B, Sharma SK. Genetic polymorphisms in TNF genes and tuberculosis in North Indians. BMC Infect Dis. 2010;10:165

127. Calhaz-Jorge C, Costa AP, Barata M. Tumour necrosis factor- $\alpha$ concentrations in the peritoneal fluid of infertile women with minimal or mild endometriosis are lower in patients with red lesions only than in patients without red lesions. Hum Reprod. 2000;15(6), 1256-60.

128. Zhang RJ, Wild RA, Ojago J. Effect of tumor necrosis factor- $\alpha$ and adhesion of human endometrial stromal cells to peritoneal mesothelial cells: an in vitro system. Fertil Steril. 1993;59(6):1196-201.

129. D'Alfonso S, Richiardi PM. A polymorphic variation in a putative regulation box of the TNFA promoter region. Immunogenetics. 1994;39(2):150-4.
130. Wilson AG, Symons JA, Mcdowell TL, McDevitt HO, Duff GW. Effects of a polymorphism in the human tumor necrosis factor alpha promoter on transcriptional activation. Proc Natl Acad Sci U S A. 1997:94(7):3195-9.

131. Bayat A, Watson JS, Stanley JK, Ferguson MW, Ollier WE. Novel single nucleotide polymorphisms in the $3^{\prime}-$ UTR of the TGF $\beta R I$ and TGF $\beta$ RIII genes. Eur J Immunogenet. 2002;29(5):445-6.

132. Sugiura $Y$, Niimi T, Sato S, Yoshinouchi T, Banno S, Naniwa T, et al. Transforming growth factor beta1 gene polymorphism in rheumatoid arthritis. Ann Rheum Dis. 2002;61(9):826-8.

133. Loverro G, Maiorano E, Napoli A, Selvaggi L, Marra E, Perlino E. Transforming growth factor-beta 1 and insulin-like growth factor-1 expression in ovarian endometriotic cysts: a preliminary study. Int J Mol Med. 2001;7:423-9.

134. Chegini N, Gold L, Willimas S. Localization of transforming growth factor $B$ isoforms TGF-BI, TGF-B2, and TGF-B3 in surgically induced endometriosis in the rat. Obstet Gynecol. 1994;83(3):455-61.

135. Oosterlynck DJ, Meuleman CR, Waer MR, Koninckx PR. Transforming growth factor- $B$ activity is increased in peritoneal fluid from women with endometriosis. Obstet Gynecol. 1994;83(2):287-92.

136. García-Velasco JA, Arici A. Interleukin-8 expression in endometrial stromal cells is regulated by integrin-dependent cell adhesion. Mol Hum Reprod.1999;5(12):1135-40.

137. Bijlsma FJ, van der Horst $A A$, Tilanus $M G$, Rozemuller $E$, de Jonge $N$, Gmelig-Meyling $\mathrm{FH}$, et al. No association between transforming growth factor beta gene polymorphism and acute allograft rejection after cardiac transplantation. Transpl Immunol. 2002;10(1):43-7. 